\title{
Endostar continuous versus intermittent intravenous infusion combined with chemotherapy for advanced NSCLC: a systematic review and meta-analysis including non-randomized studies
}

\author{
Bo Wang ${ }^{1+}$, Lu Xü ${ }^{2+}$, Qihuan Li ${ }^{1}$, Sailimai Man ${ }^{1,2}$, Cheng $\mathrm{Jin}^{3}$, Lian Liu', Siyan Zhan ${ }^{2,5^{*}}$ and Yi Ning ${ }^{1 *}$ (D)
}

\begin{abstract}
Background: Both intermittent intravenous (IIV) infusion and continuous intravenous (CIV) infusion of Endostar are widely used for NSCLC in China. We aimed to compare the efficacy and safety of CIV of Endostar versus IIV in combination with first-line chemotherapy for patients with advanced NSCLC.

Methods: RCTs, NRCTs and cohort studies which compared CIV of Endostar with IIV in advanced NSCLC patients and reported efficacy or safety outcomes were eligible. Two reviewers independently screened records, extracted data and assessed risk of bias. Pooled risk ratios (RRs) with 95\% confidence intervals were calculated using random effects meta-analysis for short-term efficacy and safety outcomes, and hazard ratios (HRs) for survival outcomes.

Results: Finally nine studies involving 597 patients were included, containing two RCTs, three NRCTs and four cohort studies. For short-term efficacy, moderate quality of evidence showed that there were no significant differences between CIV of Endostar and IIV in objective response rate (ORR; RR 1.34, 95\% Cl 0.91-1.98, $P=0.14$ ) and disease control rate (DCR; RR $1.11,95 \% \mathrm{Cl} 0.94-1.30, P=0.21$ ). Very low quality of evidence indicated that CIV of Endostar significantly improved both overall survival (OS; HR 0.69, 95\% Cl 0.48-0.99, $P=0.046$ ) and progression-free survival (PFS; HR 0.71, 95\% Cl 0.55-0.93, $P=0.01$ ) compared with IIV. As for safety outcomes, moderate quality of evidence found that CIV of Endostar significantly reduced the risk of myelosuppression (RR $0.55,95 \% \mathrm{Cl} 0.32-0.96$, $P=0.03$ ) and cardiovascular toxicity (RR $0.21,95 \% \mathrm{Cl} 0.06-0.78, P=0.02$ ) compared with IIV.

Conclusions: In advanced NSCLC, compared with IIV, CIV of Endostar had similar short-term efficacy, and substantially lower risk of myelosuppression and cardiovascular toxicity. Although very low quality of evidence supported the survival benefit of CIV compared with IIV, large RCTs with long-term follow-up are needed to demonstrate survival benefits. Caution should be given for off-label use of CIV of Endostar.
\end{abstract}

Keywords: Recombinant human endostatin, Endostar, Non-small cell lung cancer, Systematic review, Meta-analysis, Non-randomized studies

\footnotetext{
* Correspondence: Siyan-zhan@bjmu.edu.cn; ningyi_mih@163.com

${ }^{+}$Bo Wang and Lu Xu contributed equally to this work.

2Department of Epidemiology and Biostatistics, School of Public Health

Peking University, Beijing, China

'Department of Epidemiology, Meinian Institute of Health, Beijing, China

Full list of author information is available at the end of the article
}

(c) The Author(s). 2020 Open Access This article is licensed under a Creative Commons Attribution 4.0 International License, which permits use, sharing, adaptation, distribution and reproduction in any medium or format, as long as you give appropriate credit to the original author(s) and the source, provide a link to the Creative Commons licence, and indicate if changes were made. The images or other third party material in this article are included in the article's Creative Commons licence, unless indicated otherwise in a credit line to the material. If material is not included in the article's Creative Commons licence and your intended use is not permitted by statutory regulation or exceeds the permitted use, you will need to obtain permission directly from the copyright holder. To view a copy of this licence, visit http://creativecommons.org/licenses/by/4.0/ The Creative Commons Public Domain Dedication waiver (http://creativecommons.org/publicdomain/zero/1.0/) applies to the data made available in this article, unless otherwise stated in a credit line to the data. 


\section{Background}

Lung cancer is a leading cause of cancer-related deaths on a global scale. A total of 2.09 million new cases of lung cancer occurred and 1.76 million patients died of lung cancer in 2018 [1]. Lung cancer imposes heavy economic burden worldwide. In China, the annual total cost of inpatients with lung cancer increased by an average of $16.15 \%$, with the total expenditures of inpatients increasing from $\$ 2.16$ billion in 1999 to $\$ 6.33$ billion in 2005 [2]. Non-small cell lung cancer (NSCLC) accounts for around $80-85 \%$ of all cases with lung cancer. Adenocarcinoma (ADC) and squamous cell carcinoma (SqCC) are the two most common histological subtypes of NSCLC, approximately comprising $40-50 \%$ and $20-30 \%$ of all cases, respectively. Few patients with NSCLC are diagnosed at an early stage (stage I or II), at which point patients can be cured by surgical resection. Further, over $60 \%$ of patients suffered from locally advanced or metastatic lung cancer (stage III or IV) upon diagnosis [3].

For patients with advanced NSCLC, common first-line chemotherapy included docetaxel, gemcitabine, paclitaxel, vinorelbine plus cisplatin or carboplatin [4]. However, the efficacy of first-line platinum-containing therapy is limited and more effective therapies are needed. Endostatin, an angiogenesis inhibitor produced by hemangioendothelioma, was first discovered in 1997 [5]. Endostatin specifically inhibits endothelial proliferation and potently inhibits angiogenesis and tumor growth, which suggests the possibility of antiangiogenic therapy [6]. In 2005, a novel recombinant human endostatin, Recombinant Human Endostatin Injection (trade name: Endostar; code name: $\mathrm{YH}-16$ ), was approved by China's State Food and Drug Administration (SFDA) for the treatment of NSCLC [5]. Due to a nine amino acid sequence at the N-terminus (MGGSHHHHH), Endostar possessed better heat stability and proteolytic resistance compared with the endogenous protein [7].

Several studies have focused on the efficacy and safety of Endostar combined with chemotherapy versus chemotherapy alone [8-11]. A phase II, multicenter, randomized, double-blind, placebo-controlled study compared the efficacy and safety of Endostar plus paclitaxel-carboplatin (TC regimen) with placebo plus TC in advanced NSCLC patients [8]. Endostar plus TC seemed to increase objective response rate (ORR; $39.3 \%$ versus $23.0 \%, P=0.078$ ) and disease control rate (DCR; $90.2 \%$ versus $67.2 \%, P=0.004)$. However, there was no statistically significant difference in progression-free survival (PFS) or overall survival (OS) as well as the incidence of adverse events or serious adverse events between the two groups. According to a phase III, multicenter, randomized, double-blind, placebo-controlled study [9], Endostar combined with vinorelbine-cisplatin (NP regimen) enhanced the efficacy of NP regimen. In comparison with placebo plus NP, Endostar plus NP improved ORR (35.4\% versus $19.5 \%, P=0.0003$ ) and DCR (73.3\% versus $64.0 \%, P=0.035)$. Furthermore, Endostar plus NP prolonged time to progression (TTP; 6.3 months versus 3.6 months, $P<0.001$ ), OS (13.8 months versus 9.8 months, $P<0.0001)$ and increased quality of life score (QoL score; 54.4 \pm 3.7 versus 53.4 $\pm 5.9, P=$ 0.0155). Additionally, Endostar did not increase the incidence of grade $3 / 4$ adverse events. A meta-analysis [10] of 15 RCTs also confirmed that the combination of Endostar and platinum-containing two-drug chemotherapy significantly increased ORR and DCR, prolonged TTP, improved one-year survival rate and QoL, and did not increase the risk of adverse events compared with platinum-containing two-drug chemotherapy alone. Another meta-analysis [11] of prospective clinical trials found that Endostar combined with vinorelbine plus cisplatin chemotherapy (NP regimen) increased ORR and improved one-year survival rate of patients with advanced NSCLC, compared with NP regiment alone.

Traditionally, Endostar is administrated by intermittent intravenous (IIV) infusion for $3-4 \mathrm{~h}$ per day during a 14-day period, which unavoidably causes more inconvenience, affects quality of life and may reduce patient compliance. Besides, IIV also increased intravenous admixture workload of medical staff and the use of hospital beds. Since 2010, continuous intravenous (CIV) infusion via an infusion pump, which is able to deliver a variety of solutions at a constant rate for days and even weeks, has been introduced and widely off-label used in clinical practice in China. It has been argued that with this new modality for delivering a continuous infusion for days, an effective concentration of drug will be maintained and thereby the efficacy will be enhanced [12]. To our knowledge, however, the efficacy and safety of CIV versus IIV in combination with first-line chemotherapy in treating patients with advanced NSCLC have not been systematically evaluated yet. This systematic review and meta-analysis aimed to compare the two administration strategies in terms of efficacy and safety.

\section{Method}

\section{Search strategy}

We searched the following sources: 1) Electronic databases including Pubmed, Embase, CENTRAL (Cochrane Central Register of Controlled Trials), CDSR (Cochrane Database of Systematic Review), CINAHL (the Cumulative Index to Nursing and Allied Health Literature), PsycINFO and SinoMed; 2) Clinical trial registries including ClinicalTrials.gov (www.clinicaltrials.gov) and ChiCTR (Chinese Clinical Trial Registry); 3) Citation databases including Science Citation Index Expanded and CSCD (Chinese Science Citation Database); 4) CPCI (Conference proceedings including Conference Proceedings 
Citation Index) and ASCO Meeting Library; 5) Reference lists of all relevant guidelines, reviews and finally included articles; 6) Consultation with related researchers.

All databases were searched from inception to May 14, 2020 without language restriction. The keywords for the search strategy included "Endostar", "recombinant human endostatin", "Rh-endostatin" and "YH-16".

\section{Inclusion and exclusion criteria}

Eligible study types included randomized controlled trials (RCTs), non-randomized controlled trials (NRCTs) and cohort studies. Single-arm trials, case-control studies, controlled before-and-after studies, historically controlled studies, interrupted time series studies, crosssectional studies, case series, commentaries, editorials, letters, reviews, case reports and experimental studies were excluded. Eligible participants were patients with pathologically confirmed stage III or IV NSCLC, either for initial treatment or retreatment. All pathological types were eligible to be included. Eligible interventions/ controls were CIV versus IIV of Endostar in combination with first-line chemotherapy, with no limits in the dose or duration of Endostar treatment. Studies comparing Endostar combined with first-line chemotherapy versus chemotherapy alone, or those comparing Endostar combined with chemotherapy with placebo, were excluded. The study outcomes should include any of the following: 1) overall survival (OS); 2) progression-free survival (PFS); 3) ORR; 4) DCR; 5) TTP; 6) adverse events. ORR and DCR were evaluated by response evaluation criteria in solid tumors (RECIST) [13].

For studies with multiple publications or studies with same results published in different journals, the most comprehensive one with the largest sample size was chosen. Two investigators (LX and QL) independently screened the titles and abstracts of citations retrieved, and the full texts of potentially eligible articles were obtained and further assessed for final inclusion. Disagreements were resolved through consensus or consultation with a senior investigator (BW).

\section{Data extraction and risk of bias assessment}

We collected information regarding study characteristics (study design, inclusion criteria, patient characteristics, sample size, intervention and control details, outcomes, and main results) and methodological characteristics (participants recruitment, randomization, assignment concealment, blinding, intervention compliance, followup, outcome evaluation, statistical analysis).

Due to the variability of study designs included (i.e., RCTs, NRCTs and cohort studies), Downs and Black Checklist was used to assess risk of bias in each included study $[14,15]$. The items contained mainly address the following specific domains: 1) participants recruitment;
2) randomization; 3) allocation concealment; 4) blinding; 5) intervention compliance; 6) follow-up; 7) outcome assessment; 8) statistical analysis. The included 13 items can be seen in Table S1 (see Additional file 1). The possible score assigned for each item was 0 (no or unclear) and 1 (yes). The overall score ranged from 0 to 13 , with $0-5$ standing for very serious risk of bias, $6-7$ for serious risk of bias, and 8-13 for acceptable risk of bias.

Data extraction and quality evaluation were conducted by two investigators (LX and QL) in duplicate as well. Any disagreement was negotiated by the two investigators or judged by a third senior investigator (BW). Furthermore, Grading of Recommendations Assessment, Development, and Evaluation (GRADE) [16], which includes 5 aspects (study limitation, indirectness, inconsistency, imprecision and publication bias), was utilized to evaluate the quality of evidence contributing to each study outcome.

\section{Statistical analysis}

For survival outcomes (OS and PFS), hazard ratios (HRs) was chosen as the effect measure, and inverse variance method was employed to conduct the meta-analysis. For dichotomous outcomes (ORR, DCR and adverse events), risk ratios (RRs) was selected as effect measure and Mantel-Haenszel method was used for meta-analysis. In current systematic review, all the analysis were performed by using random-effect model, and were implemented and presented according to study designs (it was not appropriate to combine results from different study designs). For studies in which HR was not provided for survival outcomes, we estimated HR and its 95\% CI from Kaplan-Meier curve according to the method by Tierney et al. [17]. Meanwhile, sensitivity analysis for this approximate estimation was undertaken.

Chi-square test was used to evaluate whether there was statistically significant heterogeneity among studies, and the significance level was set to 0.10 . Heterogeneity between studies was also assessed by $\mathrm{I}^{2}$ statistic, which estimates the percentage of total variation across studies due to true between-study differences rather than chance. If there was significant heterogeneity $(P<0.10$ and $\mathrm{I}^{2} \geq 50 \%$ ), sensitivity analysis was also carried out to evaluate the robustness of results by excluding obviously outlying studies. As funnel plots should only be used if there are at least ten studies included in the metaanalysis, Harbord' test and Egger's test were implemented to identify publication bias for dichotomous and survival outcomes, respectively. Publication bias could not be detected for outcomes with less than two studies included, as the statistical tests are reliable with at least three studies included in the analysis. Statistical analyses were done using Stata 15 (Stata Corp., College Station, TX, USA). 


\section{Results}

\section{Study characteristics and risk of Bias}

Figure 1 showed the selection of eligible studies. After removing 632 duplicates, 3840 records were retrieved from literature search. Three thousand eight hundred thirty-one records were further excluded as they did not meet the inclusion criteria. Finally, nine studies were identified as eligible for inclusion in this systematic review, including four cohort studies [18-21] and two RCTs [22, 23] and three NRCTs [24-26].

Table 1 showed the characteristics of included studies. All nine studies were conducted in China, and were published between 2013 and 2019. A total of 597 advanced NSCLC participants were included (CIV: 300 and IIV: 297), with ages ranging from 24 to 78 years. The histological types of NSCLC included both SqCC and ADC in five studies [20-24], only SqCC in one study [18], and were unknown in three studies $[19,25,26]$. TNM staging of advanced NSCLC included both stage III and IV in six studies [18-20, 22-24], only stage IV in one study [26], and were not reported in two studies [21, 25]. Included studies were relatively small, with sample sizes ranging between 28 and 116. In each treatment cycle, Endostar was given at a daily dose of $7.5 \mathrm{mg} / \mathrm{m}^{2} / \mathrm{d}$, for $3-4 \mathrm{~h}$ from day 1 to 14 , in the IIV group. In the CIV group, Endostar was administered via an infusion pump $[18,21-26]$ or a mini-osmotic pump $[19,20]$, at a dose of $210 \mathrm{mg}$ with a speed of $1.8-10 \mathrm{~mL} / \mathrm{h}$ in seven studies [19-23, 25, 26], a dose of $225 \mathrm{mg}$ with a speed of $2 \mathrm{~mL} / \mathrm{h}$ in one study [24], and a dose of $135 \mathrm{mg}$ with a speed of $11 \mathrm{~mL} / \mathrm{h}$ in one study [18]. In all included studies, Endostar was administered in combination with first-line chemotherapy, and no surgery or radiotherapy was implemented. ORR, DCR and adverse events were reported in all nine studies, OS in two studies [18, 19], and PFS in four studies [18-21].

The results of risk of bias assessment can be found in Table S2 (see Additional file 1). All studies were not blinded to patients or outcome measurers. No results were based on data dredging, as no retrospective subgroup analyses were reported. In all included studies, the analyses adjusted for different lengths of follow-up of patients by survival analysis or the follow-up was the same for all participants, and appropriate statistical methods were used to assess the main outcomes. Compliance with interventions was reliable in each study, as there was no non-compliance with the allocated treatment or contamination of intervention groups. In all nine studies, participants in different intervention groups were recruited from the same population and over the same period of time, and the main outcome measures used were valid and reliable. Although patients were randomized to intervention groups in two RCTs [22, 23], we were unable to judge whether allocation was concealed from both patients and health care staff. Baseline characteristics were comparable between intervention groups in all studies but one [24], in which comparability between groups was not reported and no adjustment for confounding was conducted. None of the nine studies reported any losses of patients to follow-up. Therefore, all included studies were evaluated as with acceptable risk of bias.

\section{Efficacy outcomes}

Table 2 summarized pooled results for short-term efficacy outcomes and survival outcomes between CIV and

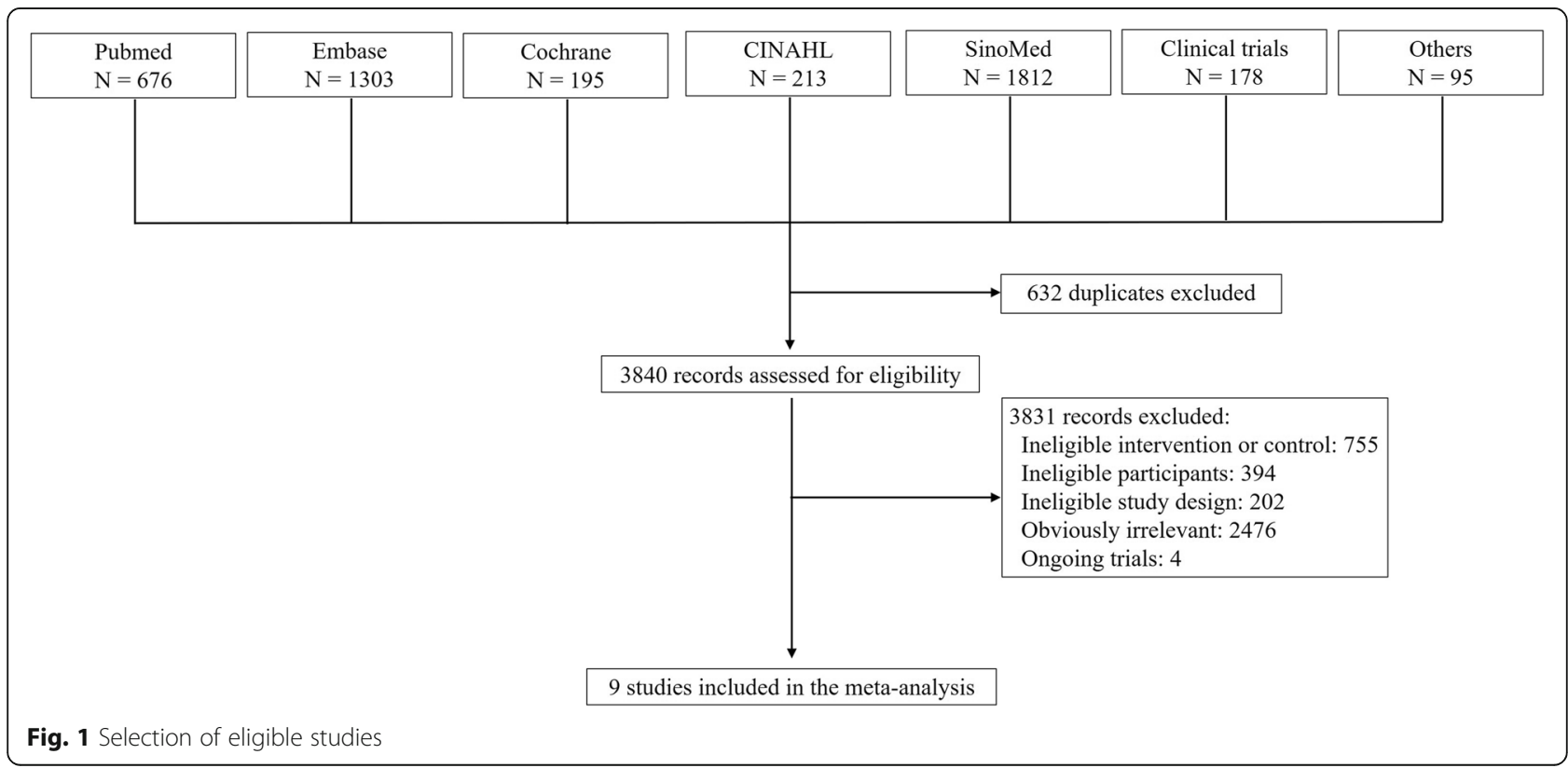




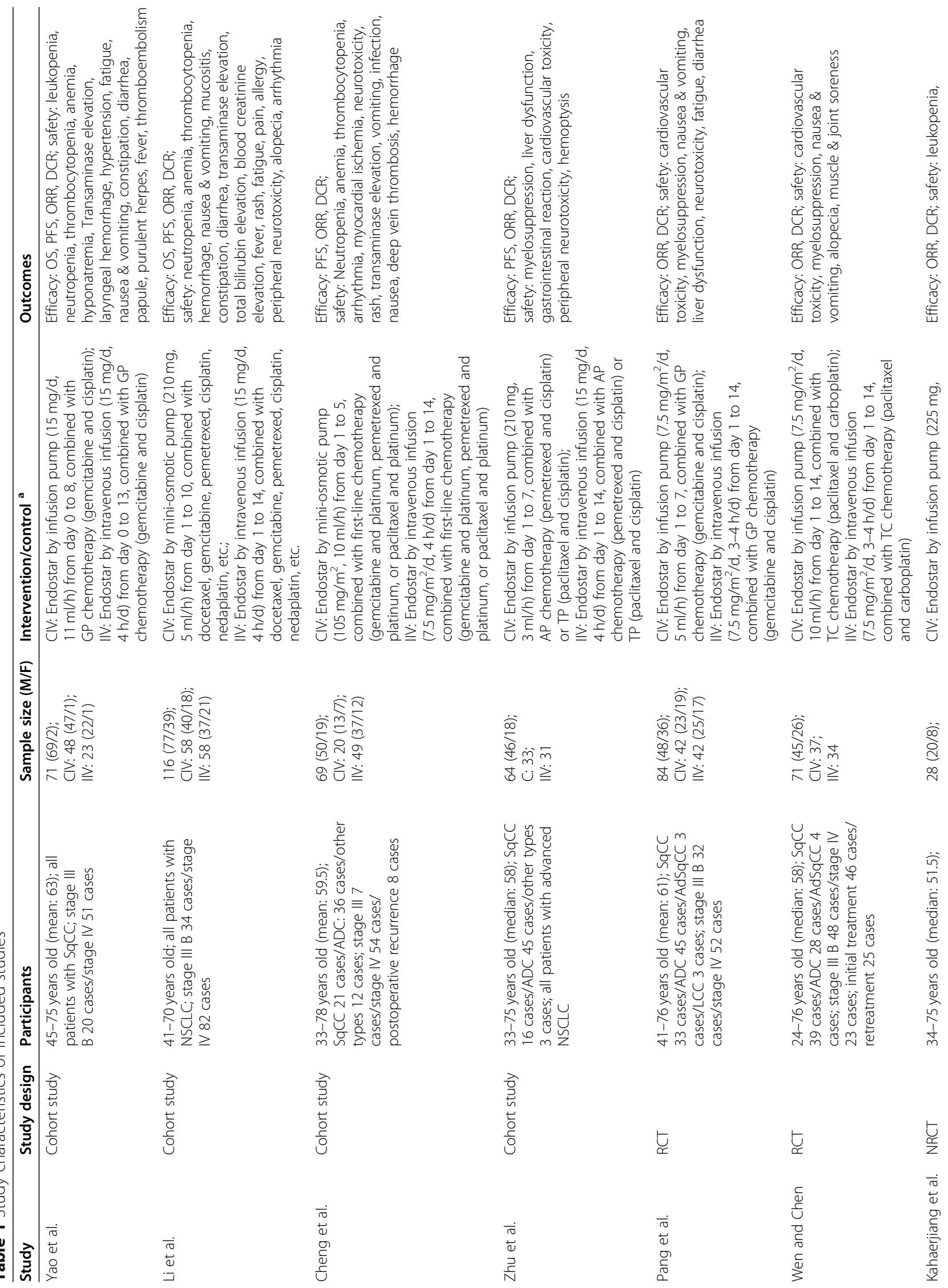


Wang et al. BMC Cancer

(2020) 20:1021

Page 6 of 14

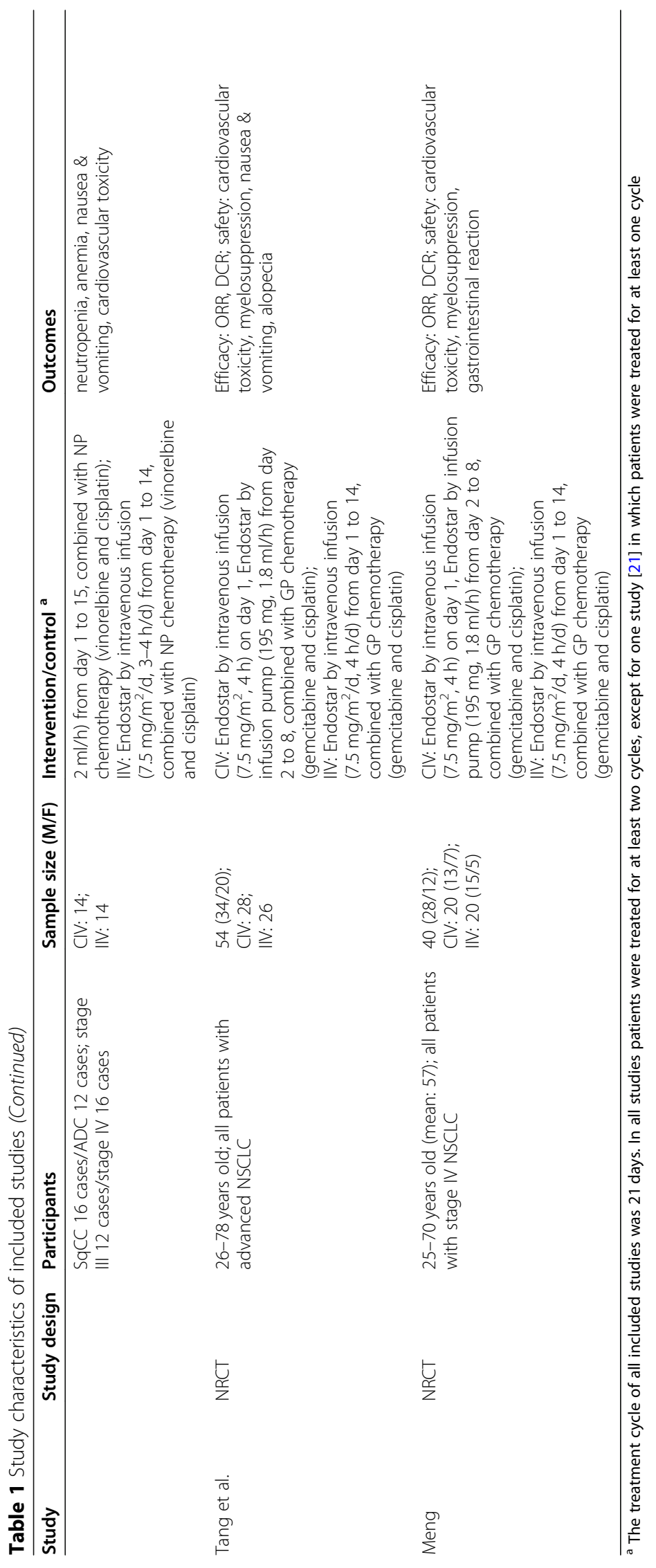


Table 2 The pooled results and evidence quality in efficacy outcomes

\begin{tabular}{|c|c|c|c|c|c|c|}
\hline \multirow[t]{2}{*}{ Outcome } & \multicolumn{2}{|l|}{$\mathrm{RCT}$} & \multicolumn{2}{|l|}{ NRCT } & \multicolumn{2}{|l|}{ Cohort study } \\
\hline & Estimate $(95 \% \mathrm{Cl})$ & Evidence quality $^{a}$ & Estimate $(95 \% \mathrm{Cl})$ & Evidence quality $^{a}$ & Estimate $(95 \% \mathrm{Cl})$ & Evidence quality $^{a}$ \\
\hline \multicolumn{7}{|c|}{ Short-term outcome } \\
\hline ORR & RR 1.34 (0.91-1.98) & Moderate & RR 1.14 (0.85-1.53) & Low & RR 1.39 (0.81-2.39) & Very low \\
\hline DCR & RR 1.11 (0.94-1.30) & Moderate & RR 1.09 (0.91-1.30) & Low & RR 1.07 (0.94-1.21) & Very low \\
\hline \multicolumn{7}{|c|}{ Survival outcome } \\
\hline OS & - & - & - & - & HR $0.68(0.42-0.94)^{*}$ & Very low \\
\hline PFS & - & - & - & - & HR $0.71(0.51-0.90)^{*}$ & Very low \\
\hline
\end{tabular}

a The evidence quality was evaluated by GRADE. Moderate: we are moderately confident in the effect estimate (the true effect is likely to be close to the estimate of the effect, but there is a possibility that it is substantially different); Low: our confidence in the effect estimate is limited (the true effect may be substantially. different from the estimate of the effect); Very low: we have very little confidence in the effect estimate (the true effect is likely to be substantially different from the estimate of effect)

${ }^{*}$ A statistically significant difference exists $(P<0.05)$

IIV of Endostar, including corresponding evidence quality in each outcome. Two RCTs [22, 23], involving 155 NSCLC patients, reported the RR of ORR for CIV compared with IIV, and the pooled RR of ORR was 1.34 (95\% CI 0.91-1.98, $P=0.14$; heterogeneity test, $\mathrm{I}^{2}=35 \%$, $P=0.22$; random-effects meta-analysis; Fig. 2). Statistical test for publication bias was not performed due to less than two included studies (similarly hereinafter). The quality of evidence was moderate. Three NRCTs [2426], including a total of 122 NSCLC patients, reported the RR of ORR and the pooled RR was 1.14 (95\% CI $0.85-1.53, P=0.37$; heterogeneity test, $\mathrm{I}^{2}=0 \%, P=0.85$; random-effects meta-analysis; Fig. 2), with low quality of evidence. Harbord's test did not indicate publication bias $(P=0.99)$. Four cohort studies [18-21], including 317 patients, reported RR of ORR and the pooled RR was 1.39 (95\% CI 0.81-2.39, $P=0.23$; heterogeneity test, $\mathrm{I}^{2}=$ $67 \%, P=0.03$; random-effects meta-analysis; Fig. 2 ), with very low quality of evidence. Harbord's test did not suggested publication bias $(P=0.43)$. Sensitivity analysis removing one obvious outlier study [21] identified no significant heterogeneity among remaining studies and did not materially change the results.

Two RCTs [22, 23], involving 155 NSCLC patients, reported the RR of DCR for CIV compared with IIV, and the pooled RR of DCR was 1.11 (95\% CI 0.94-1.30, $P=$

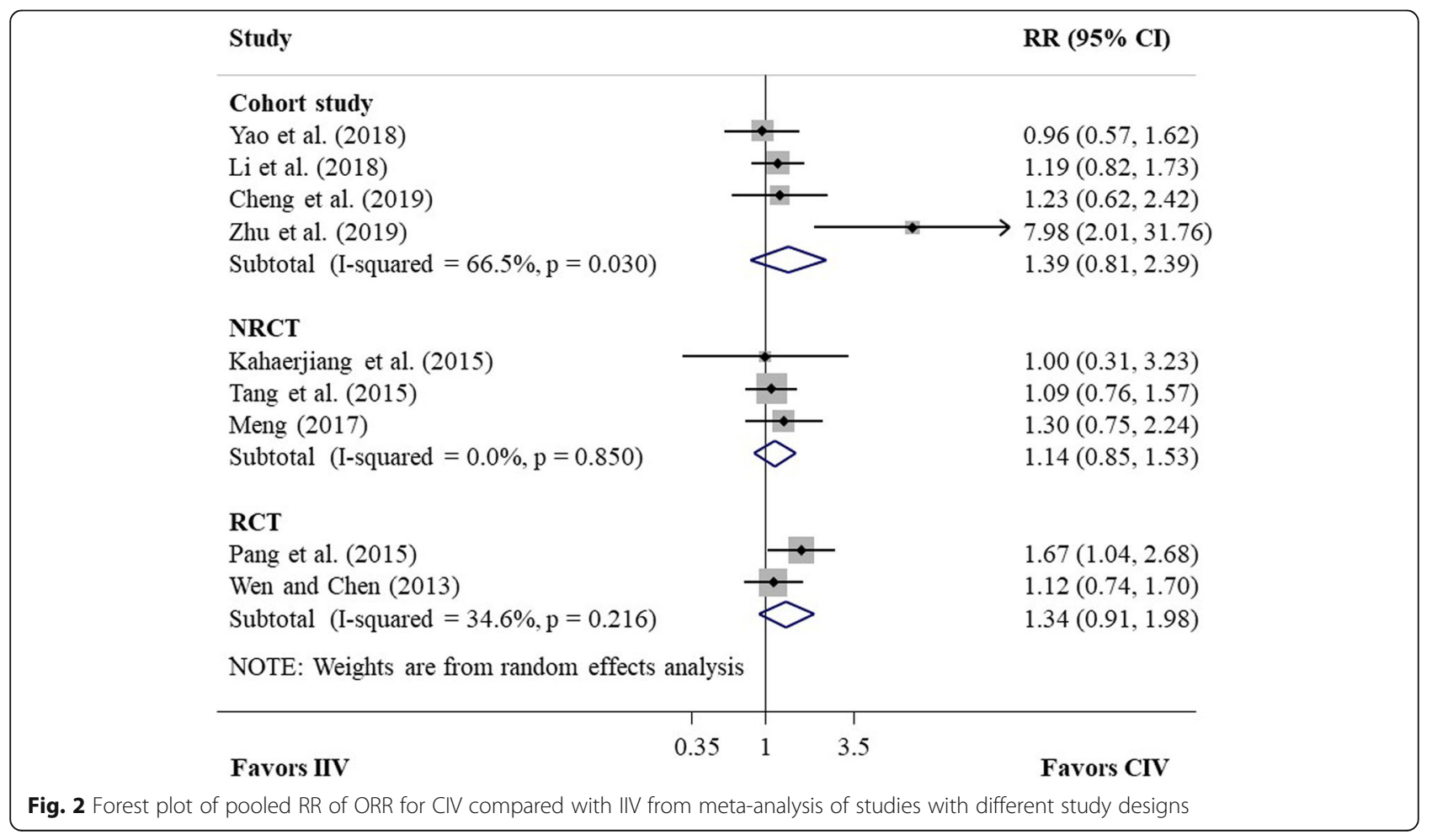


0.21 ; heterogeneity test, $\mathrm{I} 2=0 \%, P=0.33$; random-effects meta-analysis; Fig. 3). The quality of evidence was moderate. Three NRCTs [24-26], including a total of 122 NSCLC patients, reported the RR of DCR and the pooled RR was 1.09 (95\% CI 0.91-1.30, $P=0.35$; heterogeneity test, I $2=0 \%, P=0.75$; random-effects meta-analysis; Fig. 3), with low quality of evidence. Harbord's test did not find publication bias $(P=0.17)$. Four cohort studies [18-21], including 317 patients, reported RR of DCR and the pooled RR was 1.07 (95\% CI $0.94-1.21, P=0.30$; heterogeneity test, I2 = $5 \%, P=0.37$; random-effects meta-analysis; Fig. 3), with very low quality of evidence. Harbord's test suggested no publication bias $(P=0.18)$.

Two cohort studies [18, 19], involving 187 patients, provided the HR or Kaplan-Meier curve of OS for CIV compared with IIV, and the pooled HR was 0.69 (95\% CI $0.48-0.99, P=0.046$; heterogeneity test, $\mathrm{I}^{2}=0 \%, P=$ 0.60 ; random-effects meta-analysis; Fig. 4), with very low quality of evidence. Three cohort studies [18-20], including a total of 256 patients, provided the HR or Kaplan-Meier curve of PFS for CIV compared with IIV, and the pooled HR was $0.71(95 \%$ CI $0.55-0.93, P=0.01$; heterogeneity test, $\mathrm{I} 2=0 \%, P=0.83$; random-effects meta-analysis; Fig. 5), with very low quality of evidence. No publication bias was suggested by Egger's test $(P=$ 0.81 ). For both OS and PFS, sensitivity analysis, which excluded studies with approximate estimation of HR, did not materially change the direction and CIs of the results.

\section{Safety outcomes}

Table 3 exhibited the results on safety outcomes between CIV and IIV, based on meta-analysis of studies by different study designs and corresponding evidence quality in each safety outcome. The pooled results found that compared with IIV, CIV reduced the risk of myelosuppression (RR $0.55,95 \%$ CI $0.32-0.96, P=$ 0.03 ; heterogeneity test, $\mathrm{I}^{2}=58 \%, P=0.12$; randomeffects meta-analysis; moderate quality of evidence) and cardiovascular toxicity (RR $0.21,95 \%$ CI $0.06-$ $0.78, \quad P=0.02$; heterogeneity test, $\mathrm{I}^{2}=0 \%, P=0.59$; random-effects meta-analysis; moderate quality of evidence). In addition, one cohort study [18] reported that CIV reduced the risk of laryngeal hemorrhage in comparison to IIV (RR 0.27, 95\% CI 0.09-0.84, P = 0.02 ; very low quality evidence). One NRCT [25] reported that CIV reduced the risk of alopecia (RR $0.65,95 \%$ CI $0.42-1.00, P=0.05)$, however, this was not confirmed by one RCT (RR 0.95 , 95\% CI $0.82-$ $1.09, P=0.45)$. For all the other adverse events, no statistically significant differences were found between CIV and IIV of Endostar, and Harbord's test suggested no publication bias for those outcomes with at least three studies included.

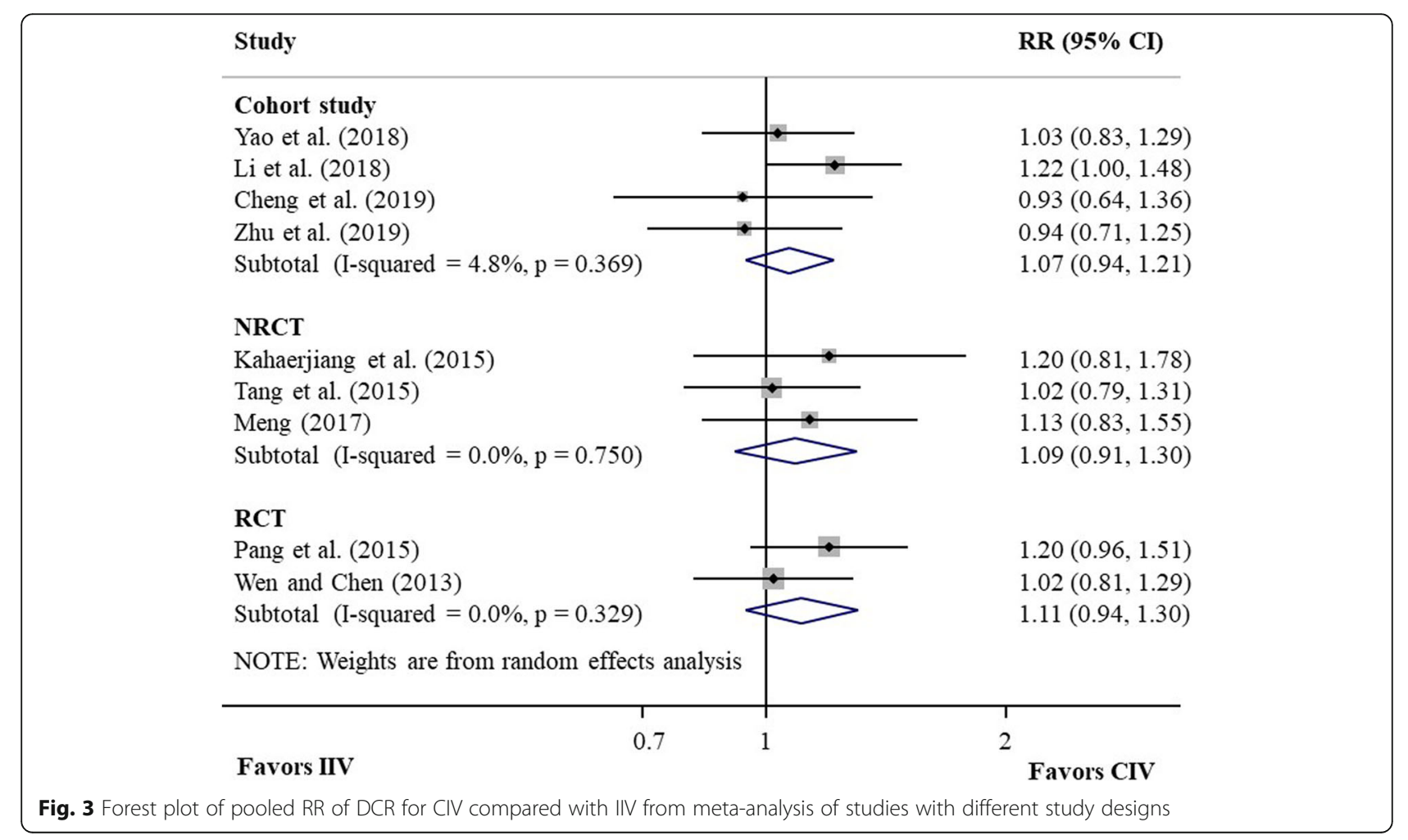




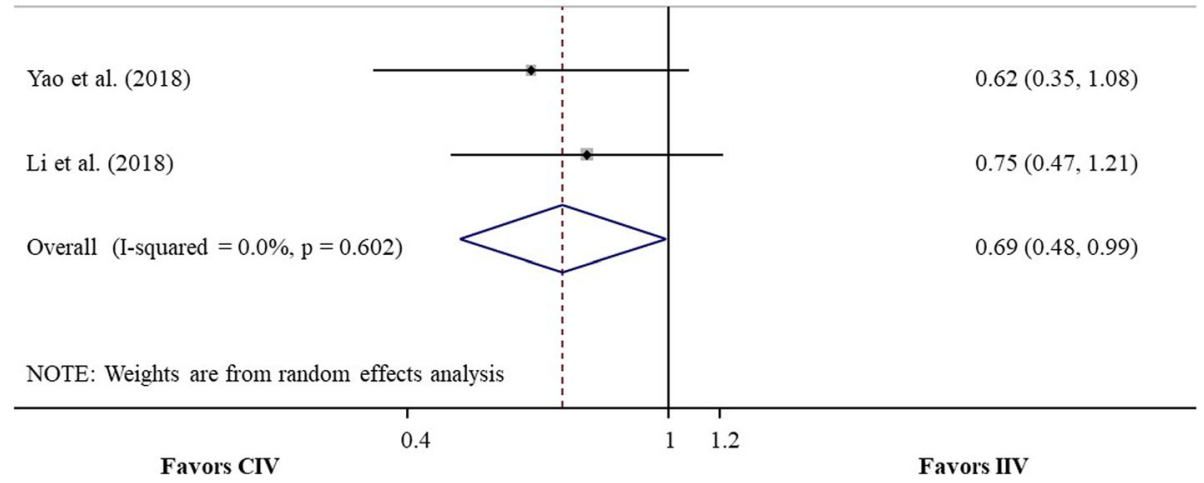

Fig. 4 Forest plot of pooled HR of OS for CIV compared with IIV from meta-analysis of cohort studies

\section{Discussion}

Proangiogenic factors are usually overexpressed in tumors, resulting in angiogenic switch. Endostatin inhibits tumor angiogenesis through a variety of angiostatic activities on endothelial cells [27]. One potential mechanism is that endostatin inhibits matrix metalloproteinases (MMPs), which mediate proteolytic degradation of the extracellular matrix that can facilitate endothelial cell migration and invasion during angiogenesis [28, 29]. Another mechanism proposes that endostatin binds to $\alpha 5$ - and $\alpha \mathrm{v}$-integrins [30, 31], with three major downstream effects: actin disassembly through Src-dependent-p190RhoGAP activation [32], inhibition of the FAK/Ras/p38-MAPK/ERK signaling cascade via $\alpha 5 \beta 1$-integrin binding [33], and downregulation of $\beta$-catenin dependent on Wnt signaling [34]. In addition, endostatin binds to vascular endothelial growth factor receptor 2 (VEGFR2) directly without binding to its ligand, inhibiting VEGF-induced phosphorylation and suppressing VEGF-mediated downstream signaling pathway $[35,36]$.

Patients diagnosed with lung cancer usually experience significant medical service utilization [37]. In clinical practice, IIV of Endostar has increased intravenous admixture workload of nursing staff, as Endostar should be diluted in $500 \mathrm{~mL}$ of normal saline daily for a duration of 14 days in each treatment cycle. In addition, NSCLC patients need to lie in bed for 3-4 h each day, hence it is difficult for them to move freely, causing more inconvenience to daily life. The CIV delivery method of Endostar can overcome aforementioned drawbacks by using a portable infusion pump, after preparation of a single admixture for each treatment cycle. Besides, the automatic pump for continuous infusion can usually accurately control the infusion rate and the amount of infusion, alarm abnormalities such as air bubbles, empty fluid, and infusion tube blockage, thus help to reduce the risk

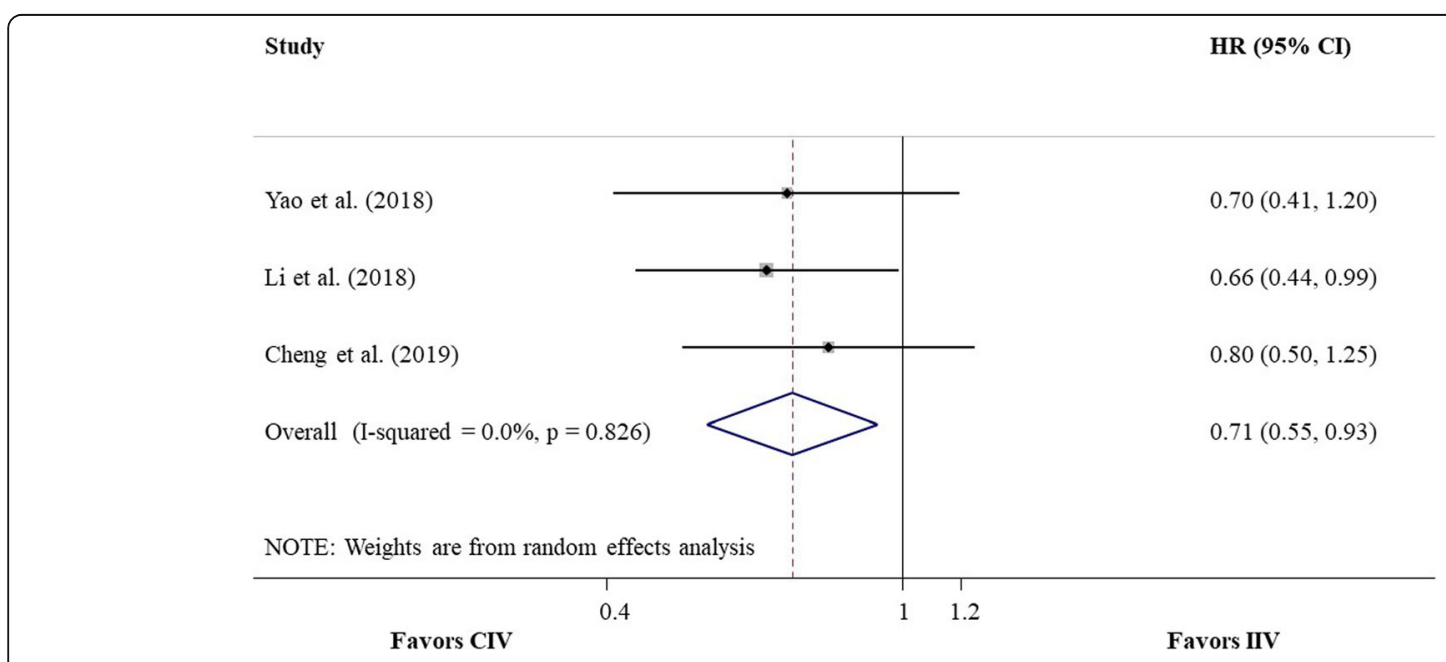

Fig. 5 Forest plot of pooled HR of PFS for CIV compared with IIV from meta-analysis of cohort studies 
Table 3 The pooled results and evidence quality in safety outcomes

\begin{tabular}{|c|c|c|c|c|c|c|}
\hline \multirow[t]{2}{*}{ Outcome } & \multicolumn{2}{|l|}{ RCT } & \multicolumn{2}{|l|}{ NRCT } & \multicolumn{2}{|l|}{ Cohort study } \\
\hline & Estimate $(95 \% \mathrm{Cl})$ & Evidence quality ${ }^{\mathrm{a}}$ & Estimate $(95 \% \mathrm{Cl})$ & Evidence quality $^{a}$ & Estimate $(95 \% \mathrm{Cl})$ & Evidence quality $^{a}$ \\
\hline Myelosuppression & RR $0.55(0.32-0.96)^{*}$ & Moderate & RR $0.38(0.20-0.72)^{*}$ & Low & RR $1.11(0.59-2.10)$ & Very low \\
\hline $\begin{array}{l}\text { Grade } 3 / 4 \\
\text { myelosuppression }\end{array}$ & RR $0.70(0.29-1.66)$ & Low & RR $0.20(0.01-3.92)$ & Very low & RR $1.25(0.30-5.15)$ & Very low \\
\hline Leukopenia & - & - & RR $0.44(0.18-1.11)$ & Very low & RR $1.12(0.79-1.58)$ & Very low \\
\hline Grade 3/4 leukopenia & - & - & - & - & RR $0.80(0.33-1.93)$ & Very low \\
\hline Neutropenia & - & - & RR $0.55(0.28-1.06)$ & Very low & RR 1.04 (0.89-1.22) & Very low \\
\hline Grade 3/4 neutropenia & - & - & - & - & RR $0.96(0.64-1.43)$ & Very low \\
\hline Anemia & - & - & RR $0.43(0.14-1.33)$ & Very low & RR $1.05(0.92-1.20)$ & Very low \\
\hline Grade 3/4 anemia & - & - & - & - & RR $0.95(0.46-1.97)$ & Very low \\
\hline Thrombocytopenia & - & - & - & - & RR 1.15 (0.74-1.79) & Very low \\
\hline Grade 3/4 thrombocytopenia & - & - & - & - & RR $1.30(0.49-3.47)$ & Very low \\
\hline Hemorrhage & - & - & - & - & - & - \\
\hline Grade 3/4 hemorrhage & - & - & - & - & - & - \\
\hline Laryngeal hemorrhage & - & - & - & - & $\operatorname{RR} 0.27(0.09-0.84)^{*}$ & Very Low \\
\hline Cardiovascular toxicity & RR $0.21(0.06-0.78)^{*}$ & Moderate & RR $0.15(0.02-1.20)$ & Very low & - & - \\
\hline Cardiotoxicity & - & - & RR $0.26(0.06-1.13)$ & Very low & - & - \\
\hline Gastrointestinal Reaction & - & - & RR $0.83(0.30-2.29)$ & Very low & RR $1.25(0.30-5.15)$ & Very low \\
\hline $\begin{array}{l}\text { Grade } 3 / 4 \text { gastrointestinal } \\
\text { response }\end{array}$ & - & - & RR $0.67(0.12-3.57)$ & Very low & - & - \\
\hline Nausea \& vomiting & RR $0.88(0.51-1.51)$ & Low & RR $0.58(0.25-1.36)$ & Very low & RR $0.97(0.72-1.31)$ & Very low \\
\hline $\begin{array}{l}\text { Grade } 3 / 4 \text { nausea \& } \\
\text { vomiting }\end{array}$ & RR $1.00(0.38-2.60)$ & Low & RR $1.00(0.16-6.14)$ & Very low & RR $1.25(0.35-4.42)$ & Very low \\
\hline Nausea & - & & - & - & RR $1.17(0.32-4.28)$ & Very low \\
\hline Vomiting & - & & - & - & RR $1.23(0.63-2.40)$ & Very low \\
\hline Diarrhea & RR $1.43(0.60-3.40)$ & Low & - & - & RR $0.60(0.18-1.96)$ & Very low \\
\hline Constipation & - & & - & - & RR $0.90(0.53-1.54)$ & Very low \\
\hline Alopecia & RR 0.95 (0.82-1.09) & Moderate & RR $0.65(0.42-1.00)^{*}$ & Very low & RR $0.78(0.31-1.95)$ & Very low \\
\hline Neurotoxicity & RR $0.80(0.23-2.77)$ & Low & - & - & - & - \\
\hline Peripheral neurotoxicity & - & & - & - & RR 0.58 (0.14-2.33) & Very low \\
\hline Liver dysfunction & RR $0.71(0.39-1.29)$ & Low & - & - & RR $0.63(0.20-2.01)$ & Very low \\
\hline Grade 3/4 liver dysfunction & RR $0.60(0.15-2.35)$ & Low & - & - & - & \\
\hline Transaminase elevation & - & & - & - & RR $1.48(0.84-2.62)$ & Very low \\
\hline Fatigue & RR $1.14(0.87-1.50)$ & Moderate & - & - & RR $0.90(0.64-1.27)$ & Very low \\
\hline Grade 3/4fatigue & RR $1.17(0.43-3.18)$ & Low & - & - & - & - \\
\hline Muscle \& joint soreness & RR $0.71(0.30-1.71)$ & Low & - & - & - & - \\
\hline Rash & - & - & - & - & RR 0.61 (0.10-3.73) & Very low \\
\hline Papule and purulent herpes & - & - & - & - & RR $1.60(0.49-5.25)$ & Very low \\
\hline Fever & - & - & - & - & RR 0.95 (0.48-1.88) & Very low \\
\hline Thromboembolism & - & - & - & - & RR $0.48(0.07-3.19)$ & Very low \\
\hline Hyponatremia & - & - & - & - & RR $1.28(0.71-2.29)$ & Very low \\
\hline Grade 3/4 hyponatremia & - & - & - & - & RR 0.48 (0.10-2.19) & Very low \\
\hline Pain & - & - & - & - & RR $1.00(0.37-2.67)$ & Very low \\
\hline
\end{tabular}

${ }^{a}$ The evidence quality was evaluated by GRADE. Moderate: we are moderately confident in the effect estimate (the true effect is likely to be close to the estimate of the effect, but there is a possibility that it is substantially different); Low: our confidence in the effect estimate is limited (the true effect may be substantially different from the estimate of the effect); Very low: we have very little confidence in the effect estimate (the true effect is likely to be substantially different from the estimate of effect)

${ }^{*}$ A statistically significant difference exists $(P<0.05)$ 
of medical errors [25]. Currently, both CIV and IIV of Endostar are administered for hospital inpatients in clinical practice in China. As seen in our systematic review, the duration of administration by CIV was usually shorter (less than 10 days in most studies) than II (usually 14 days in one treatment cycle). Two studies [18, 25] confirmed that CIV of Endostar reduced total volume of infusion, and shortened hospital stay.

Our scoping search before this systematic review indicated that a very small number of potentially relevant RCTs had been conducted previously, and survival outcomes or safety outcomes were poorly addressed. Furthermore, the role of post-approval observational studies in comparative effectiveness research has gained increasing attention in recent years. Therefore, it had been decided to conduct a systematic review and meta-analysis including both RCTs and non-randomized studies, to compare the efficacy and safety of different delivery methods of Endostar. Specifically, NRCTs and cohort studies were included as they are more likely to provide unbiased results than other non-randomized study designs. The inclusion of non-randomised studies have posed several challenges for the design and conduct of this systematic review. First, non-randomized studies are usually poor indexed with inconsistent use of study design labels [38], therefore, one comprehensive search strategy was applied in this systematic review to avoid missing any eligible studies. The second challenge arose in the assessment of risk of bias, as different study designs were included. Downs and Black Checklist can be used to evaluate both randomized and non-randomized studies. Additionally, it was considered to be one of the most valuable tools for evaluating the quality of nonrandomized studies [15]. There are 27 items in the checklist distributed between four scales: reporting (ten items), external validity (three items), internal validity (thirteen items) and power (one item). Only the internal validity scale was used for assessing the risk of bias, as generally external validity, reporting or power are unlikely to have direct implications for risk of bias [38]. Third, evidence from NRCTs were not clearly considered or included in the GRADE approach [16]. To be conservative, NRCTs without special strengths or important limitations were judged to provide low quality evidence in this systematic review, as cohort studies were. Fourth, it was possible that different qualities of evidence might have resulted from different study designs addressing the same outcome, as GRADE approach was performed according to different types of study. When this happened (which could be seen for shortterm efficacy outcomes in Table 2 and several adverse outcomes in Table 3), estimates from the highest level of quality were adopted and reported as the best available evidence, based on epistemological rationale of evidence-based medicine and principles of GRADE system $[16,39]$. Usually higher hierarchy of evidence comes from pooled results of randomized controlled studies, however, there are times when high confidence has been attached to the estimates of effect from non-randomized studies (depending on quality rating results) [40].

This systematic review and meta-analysis found no significant differences between CIV and IIV in short-term efficacy outcomes (ORR and DCR). In terms of survival outcomes, however, very low quality of evidence supported the survival benefit associated with CIV in both OS and PFS compared with IIV. The half-life of Endostar in human body is only about $10 \mathrm{~h}$ [41]. Theoretically, IIV can make the concentration of Endostar fluctuate greatly, which means the effective concentration can only act on the tumor tissue in a short time. Instead, CIV can realize the delivery of various solutions or suspensions at a constant rate for days and even weeks maintaining the effective concentration of Endostar [12], which may explain why CIV of Endostar might improve survival outcomes over IIV. Large RCTs with long-term follow-up are needed to definitely demonstrate the efficacy of CIV in comparison with IIV of Endostar.

As for safety outcomes, moderate quality of evidence demonstrated that compared with IIV, CIV of Endostar reduced the risk of myelosuppression (RR $0.55,95 \% \mathrm{CI}$ $0.32-0.96$ ) and cardiovascular toxicity (RR $0.21,95 \% \mathrm{CI}$ $0.06-0.78$ ) by 45 and $79 \%$, respectively. Especially, the reduction in the risk of cardiovascular toxicity was substantial. Very low quality evidence suggested that CIV might decrease the risk of laryngeal hemorrhage (RR 0.27, 95\% CI 0.09-0.84). For all the other adverse events (including all the grade $3 / 4$ adverse reactions), the vast majority of evidence was of low or very low quality, and identified no differences between CIV and IIV. Proteinuria, injection site-related adverse events and phlebitis were not investigated in this systematic review, as they were reported in none of the included studies. It is speculated that by adopting CIV, the amount of drug pumped per unit time is less and the fluctuation of drug concentration is mild, thus it has probably less adverse impact on bone marrow and cardiovascular system. Previous studies also supported that CIV of Endostar could reduce drug toxicity [41].

Lung cancer is one of the major contributors to cancer-caused DALYs in most countries, and has imposed a substantial disease burden to global public health $[42,43]$. China has experienced a noteworthy increase in the relative disease burden caused by lung cancer, with $12 \%$ of total DALYs from cancers in 1990 to $20 \%$ in 2008 [43], and is now facing up with severe predicament of lung cancer burden [37]. Currently, Endostar has been widely used in combination with first-line 
chemotherapy for advanced NSCLC in China. Its use has been recommended by National Health Commission of China [44] and Chinese Society of Clinical Oncology [45], and has been covered by National Health Insurance of China. With the increasing offlabel use of CIV of Endostar in treatment of NSCLC, it is essential to get better knowledge of the relative efficacy and safety of CIV versus IIV to guide clinical practice. Our study has provided current best available evidence for this important clinical question. With scientific and rigorous methods employed, the results indicated that CIV had similar short-term efficacy and lower risk of certain adverse outcomes, and suggested possible survival benefit compared with IIV. Given the fact that survival benefit associated with CIV has not substantiated by high quality of evidence, we argue that advisable caution should be given in clinical off-label use of CIV of Endostar. Future large RCTs with sufficient follow-up, or at least welldesigned and executed real-world studies, are warranted to demonstrate the relative survival benefit of CIV.

Our review has three advantages. First, to the best of our knowledge, this is the first systematic review and meta-analysis head-to-head comparing the efficacy and safety of CIV of Endostar with IIV, and the results will inform clinical decision-making in NSCLC treatment. Second, this systematic review included both RCTs and non-randomized studies (NRCTs and cohort studies), and provided an overall evidence profile on the benefit and harm of different delivery methods of Endostar in the treatment of NSCLC. Third, a comprehensive search strategy with high sensitivity was performed, to accommodate the inclusion of non-randomized studies and conduct a search as exhaustive as possible. Our review also suffers from several limitations. First, with a relatively small number of participants in each outcome, imprecision of effect estimates had led to downgrading one or two levels in quality of evidence in all outcomes. Second, due to the small number of studies included (and analysis was not implemented by different histological types in all primary studies), subgroup analysis or meta-regression were not carried out to investigate the influence of histological type, dose, infusion rate and TNM staging on the pooled results. Third, baseline comparability of EGFR status was not assessed or adjusted in most included studies, and this could lead to potential confounding.

\section{Conclusions}

In conclusion, CIV and IIV of Endostar, in combination with first-line chemotherapy, had similar short-term efficacy in patients with pathologically confirmed stage III or IV NSCLC. Compared with IIV, CIV of Endostar reduced the risk of myelosuppression and cardiovascular toxicity substantially. Very low quality evidence supported that CIV could improve both OS and PFS, and large RCTs with long-term follow-up are needed to demonstrate these survival benefits. Advisable caution should be given for off-label use of CIV of Endostar in clinical practice.

\section{Supplementary information}

The online version contains supplementary material available at https://doi. org/10.1186/s12885-020-07527-4

Additional file 1.

\section{Abbreviations}

ADC: Adenocarcinoma; CDSR: Cochrane Database of Systematic Review; CENTRAL: Cochrane Central Register of Controlled Trials; CIV: Continuous intravenous; CPCl: Conference proceedings including Conference

Proceedings Citation Index; DALYs: Disability adjusted life years; DCR: Disease control rate; HRs: Hazard ratios; IIV: Intermittent intravenous; MMPs: Matrix metalloproteinases; NRCTs: Non-randomized controlled trials; NSCLC: Nonsmall cell lung cancer; ORR: Objective response rate; OS: Overall survival; FS: Progression-free survival; RCTs: Randomized controlled trials; RECl ST: Response evaluation criteria in solid tumors; RRs: Risk ratios; SFDA: State Food and Drug Administration; SqCC: Squamous cell carcinoma; TTP: Time to progression; QoL: Quality of life; VEGRF2: Vascular endothelial growth factor receptor 2

\section{Acknowledgements}

Not applicable.

Availability of data and materials (data transparency)

All data generated or analysed during this systematic review are available from published literatures.

\section{Authors' contributions}

All authors have read and approved the manuscript. BW contributed to the study conception, performed the search, and drafted the manuscript. LX performed the data extraction, assessed the risk of bias of the studies, and drafted the manuscript. QL performed the data extraction and assessed the risk of bias of the studies. SM performed the search, conducted the statistical analyses, and reviewed the manuscript for important intellectual content. CJ conducted the statistical analyses and reviewed the manuscript for important intellectual content. LL contributed to the study conception, supervised the statistical analyses, and reviewed the manuscript for important intellectual content. SZ contributed to the study conception, supervised the project, and reviewed the manuscript for important intellectual content. YN contributed to the study conception, supervised the project, and reviewed the manuscript for important intellectual content.

\section{Funding}

This study was partially funded by Jiangsu Simcere Pharmaceutical Co. Ltd (Jiangsu, China). However, the funding source had no role in the design, conduct or analysis of this systematic review, or the decision to submit results.

Ethics approval and consent to participate Not applicable.

Consent for publication

Not applicable.

\section{Competing interests}

The authors meet the criteria for authorship as recommended by the International Committee of Medical Journal Editors. The authors received no 
direct compensation related to the development of the manuscript. The authors declare that they have no conflicts of interest.

\section{Author details}

${ }^{1}$ Department of Epidemiology, Meinian Institute of Health, Beijing, China. ${ }^{2}$ Department of Epidemiology and Biostatistics, School of Public Health, Peking University, Beijing, China. ${ }^{3}$ Department of Biostatistics, Meinian Institute of Health, Beijing, China. ${ }^{4}$ State Key Laboratory of Translational Medicine and Innovative Drug Development, Nanjing, Jiangsu, China. ${ }^{5}$ Research Center of Clinical Epidemiology, Peking University Third Hospital, Beijing, China.

Received: 26 June 2020 Accepted: 14 October 2020 Published online: 21 October 2020

\section{References}

1. International Agency for Research on Cancer. Cancer Today: Fact sheets. 2019. http://gco.iarc.fr/today/data/factsheets/cancers/15-Lung-fact-sheet.pdf. Accessed 20 Mar, 2020.

2. She J, Yang P, Hong Q, Bai C. Lung cancer in China: challenges and interventions. Chest. 2013;143(4):1117-26.

3. Osmani L, Askin F, Gabrielson E, Li QK. Current WHO guidelines and the critical role of immunohistochemical markers in the subclassification of nonsmall cell lung carcinoma (NSCLC): moving from targeted therapy to immunotherapy. Semin Cancer Biol. 2018;52(Pt 1):103-9.

4. Pilkington $G$, Boland A, Brown T, Oyee J, Bagust A, Dickson R. A systematic review of the clinical effectiveness of first-line chemotherapy for adult patients with locally advanced or metastatic non-small cell lung cancer. Thorax. 2015;70(4):359-67.

5. Ling $Y$, Yang $Y$, Lu N, You QD, Wang $S$, Gao $Y$, et al. Endostar, a novel recombinant human endostatin, exerts antiangiogenic effect via blocking VEGF-induced tyrosine phosphorylation of KDR/FIk-1 of endothelial cells. Biochem and Biophys Res Commun. 2007;361(1):79-84

6. O'Reilly MS, Boehm T, Shing Y, Fukai N, Vasios G, Lane WS, et al. Endostatin: an endogenous inhibitor of angiogenesis and tumor growth. Cell. 1997; 88(2):277-85.

7. Huang R, Zhan Q, Zhou X, Chu Z, Jiang J, Liang X. Continuous administration of recombinant human endostatin (Endostar): a pre-clinical safety study. Exp Ther Med. 2012;3(6):1018-22.

8. Han B, Xiu Q, Wang H, Shen J, Gu A, Luo Y, et al. A multicenter, randomized, double-blind, placebo-controlled study to evaluate the efficacy of paclitaxel-carboplatin alone or with endostar for advanced non-small cell lung cancer. J Thorac Oncol. 2011;6(6):1104-9.

9. Sun Y, Wang JW, Liu YY, Yu QT, Zhang YP, Li K, et al. Long-term results of a randomized, double-blind, and placebo-controlled phase III trial: Endostar (rh-endostatin) versus placebo in combination with vinorelbine and cisplatin in advanced non-small cell lung cancer. Thorac Cancer. 2013:4(4):440-8

10. Rong B, Yang S, Li W, Zhang W, Ming Z. Systematic review and metaanalysis of Endostar (rh-endostatin) combined with chemotherapy versus chemotherapy alone for treating advanced non-small cell lung cancer. World J Surg Oncol. 2012;10:170.

11. An J, Lv W. Endostar (rh-endostatin) versus placebo in combination with vinorelbine plus cisplatin chemotherapy regimen in treatment of advanced non-small cell lung cancer: a meta-analysis. Thorac Cancer. 2018;9(5):606-12.

12. Hansma AH, Broxterman HJ, van der Horst I, Yuana Y, Boven E, Giaccone G, et al. Recombinant human endostatin administered as a 28-day continuous intravenous infusion, followed by daily subcutaneous injections: a phase I and pharmacokinetic study in patients with advanced cancer. Ann Oncol. 2005;16(10):1695-701.

13. Schwartz LH, Litiere $S$, de Vries E, Ford R, Gwyther S, Mandrekar S, et al. RECl ST 1.1-update and clarification: from the RECIST committee. Eur J Cancer. 2016:62:132-7.

14. Downs SH, Black N. The feasibility of creating a checklist for the assessment of the methodological quality both of randomised and non-randomised studies of health care interventions. J Epidemiol Community Health. 1998; 52(6):377-84.

15. Deeks JJ, Dinnes J, D'Amico R, Sowden AJ, Sakarovitch C, Song F, et al. Evaluating non-randomised intervention studies. Health Technol Assess. 2003;7(27):iii-X, 1-173.
16. Guyatt G, Oxman AD, Akl EA, Kunz R, Vist G, Brozek J, et al. GRADE guidelines: 1. Introduction-GRADE evidence profiles and summary of findings tables. J Clin Epidemiol. 2011;64(4):383-94.

17. Tierney JF, Stewart LA, Ghersi D, Burdett S, Sydes MR. Practical methods for incorporating summary time-to-event data into meta-analysis. Trials. 2007;8(1):16.

18. Yao D, Shen H, Huang J, Yuan Y, Dai H. Influence of different drug delivery methods for Endostar combined with a gemcitabine/cisplatin regimen in locally advanced or metastatic lung squamous cell carcinoma: a retrospective observational study. Medicine. 2018:97(32):e11822.

19. Li X, Gu G, Soliman F, Sanders AJ, Wang X, Liu C. The evaluation of durative transfusion of Endostar combined with chemotherapy in patients with advanced non-small cell lung Cancer. Chemotherapy. 2018;63(4):214-9. https://doi.org/10.1159/000493098.

20. Cheng Y, Nie L, Liu Y, Jin Z, Wang X, Hu Z. Comparison of Endostar continuous versus intermittent intravenous infusion in combination with first-line chemotherapy in patients with advanced non-small cell lung cancer. Thorac Cancer. 2019;10(7):1576-80.

21. Zhu F, Tang XF, Sun JB. Observation and nursing of different administration methods of Endostar combined with chemotherapy for advanced nonsmall cell lung cancer. Fujian Med J. 2019;41(1):156-8.

22. Pang LR, Chen J, Lu JE, Huang J, Li H, Xu CH, Zheng HY. Clinical study of different administration methods of recombinant human endostatin combined with GP regimen in the treatment of advanced non-small cell lung cancer. Modern Practical Medicine. 2015;27(2):255-6 276.

23. Wen XP, Chen JH. Different administration ways of Endostar combined with TC regimen in advanced non-small cell lung Cancer patients. The World's Latest Medical Information Abstracts. 2013;13(12):5-8.

24. Kahaerjiang A, Wang XL, Liu CL. Clinical study on different administration of Endostar combined with NP regimen in treatment of 28 advanced nonsmall cell lung cancer. Xinjiang Med J. 2015;45(12):1762-5.

25. Tang YQ, Ma HM, Wan YH. Clinical observation of continuous pumping of Endostar combined with GP chemotherapy for non-small cell lung cancer. Chinese Med Equipment J. 2015;36(10):71-90.

26. Meng M. Efficacy of two ways of infusion of Endostar combined with chemotherapy on advanced non small cell lung cancer. Clin Med. 2017; 37(11):28-30.

27. Poluzzi C, lozzo RV, Schaefer L. Endostatin and endorepellin: a common route of action for similar angiostatic cancer avengers. Adv Drug Deliv Rev. 2016;97:156-73.

28. Deryugina El, Quigley JP. Tumor angiogenesis: MMP-mediated induction of intravasation- and metastasis-sustaining neovasculature. Matrix Biol. 2015; 44-46:94-112

29. Shay G, Lynch CC, Fingleton B. Moving targets: Emerging roles for MMPs in cancer progression and metastasis. Matrix Biol. 2015;44-46:200-6.

30. Sudhakar A, Sugimoto H, Yang C, Lively J, Zeisberg M, Kalluri R. Human tumstatin and human endostatin exhibit distinct antiangiogenic activities mediated by alpha $\vee$ beta 3 and alpha 5 beta 1 integrins. Proc Natl Acad Sci U S A. 2003;100(8):4766-71.

31. Shichiri M, Hirata Y. Antiangiogenesis signals by endostatin. FASEB J. 2001; 15(6):1044-53.

32. Wickstrom SA, Alitalo K, Keski-Oja J. Endostatin associates with integrin alpha5beta1 and caveolin-1, and activates Src via a tyrosyl phosphatase-dependent pathway in human endothelial cells. Cancer Res. 2002;62(19):5580-9.

33. Wickstrom SA, Veikkola T, Rehn M, Pihlajaniemi T, Alitalo K, Keski-Oja J. Endostatin-induced modulation of plasminogen activation with concomitant loss of focal adhesions and actin stress fibers in cultured human endothelial cells. Cancer Res. 2001;61(17):6511-6.

34. Hanai J, Gloy J, Karumanchi SA, Kale S, Tang J, Hu G, et al. Endostatin is a potential inhibitor of Wnt signaling. J Cell Biol. 2002;158(3):529-39.

35. Kim YM, Hwang S, Kim YM, Pyun BJ, Kim TY, Lee ST, et al. Endostatin blocks vascular endothelial growth factor-mediated signaling via direct interaction with KDR/Flk-1. J Biol Chem. 2002;277(31):27872-9.

36. Eriksson K, Magnusson P, Dixelius J, Claesson-Welsh L, Cross MJ. Angiostatin and endostatin inhibit endothelial cell migration in response to FGF and VEGF without interfering with specific intracellular signal transduction pathways. FEBS Lett. 2003;536(1-3):19-24.

37. Ding R, Zhu D, He P, Ma Y, Chen Z, Shi X. Comorbidity in lung cancer patients and its association with medical service cost and treatment choice in China. BMC Cancer. 2020;20:250. 
38. The Cochrane Collaboration. Cochrane Handbook for Systematic Reviews of Interventions Version 5.1.0. 2011. https://training.cochrane.org/handbook. Accessed 20 Mar, 2020.

39. Murad MH, Asi N, Alsawas M, Alahdab F. New evidence pyramid. Evid Based Med. 2016;21(4):125-7.

40. Schünemann H, Brozek J, Guyatt G, Oxman A. Grade Handbook: handbook for grading the quality of evidence and the strength of recommendation using the GRADE approach. Updated September 2013. https://gdt.gradepro.org/app/ handbook/handbook.html\#h.svwngs6pm0f2. Accessed 8 Sept, 2020.

41. Sim BK, Fogler WE, Zhou XH, Liang H, Madsen JW, Luu K, et al. Zinc liganddisrupted recombinant human Endostatin: potent inhibition of tumor growth, safety and pharmacokinetic profile. Angiogenesis. 1999;3(1):41-51.

42. Torre LA, Freddie B, Siegel RL, Jacques F, Joannie LT, Ahmedin J. Global cancer statistics, 2012. Ca A Cancer J Clinicians. 2015;65(2):69-90.

43. Soerjomataram I, Lortet-Tieulent J, Parkin DM, Ferlay J, Mathers C, Forman D, et al. Global burden of cancer in 2008: a systematic analysis of disabilityadjusted life-years in 12 world regions. Lancet. 2012;380(9856):1840-50.

44. National Health Commission of China. Chinese guidelines for diagnosis and treatment of primary lung cancer 2018 (English version). Chin J Cancer Res. 2019;31(1):1-28

45. The Chinese Society of Clinical Oncology (CSCO). CSCO clinical guidelines for the diagnosis and treatment of primary lung cancer (2018): China People's medical publishing house co. Ltd.; 2019.

\section{Publisher's Note}

Springer Nature remains neutral with regard to jurisdictional claims in published maps and institutional affiliations.

Ready to submit your research? Choose BMC and benefit from:

- fast, convenient online submission

- thorough peer review by experienced researchers in your field

- rapid publication on acceptance

- support for research data, including large and complex data types

- gold Open Access which fosters wider collaboration and increased citations

- maximum visibility for your research: over $100 \mathrm{M}$ website views per year

At BMC, research is always in progress.

Learn more biomedcentral.com/submissions 